\title{
THE MODEL OF FOREIGN BUYERS' ANALYSIS BASED ON BELLMAN-ZADEH APPROACH UNDER THE GLOBAL MARKET UNCERTAINTY
}

\author{
O. Martyanova ${ }^{1}$ \\ DOI: http://doi.org/10.15350/L_26/10/1
}

\section{Abstract}

The paper provides methodological toolkit for evaluating foreign buyers based on Bellman-Zadeh approach, enabling to carry out a multiple criteria analysis of the set in order to choose the best alternative that simultaneously meets all the criteria and constraints to the maximum extent.

Keywords: multiple criteria analysis, fuzzy set, Bellman-Zadeh approach, optimal alternative, analytic hierarchy process, paired comparison matrix, membership function, importance coefficient, relative importance of criteria, consistency index, eigenvector.

Decision making in the sphere of international trade is currently carried out under the conditions when the international trade determined by the existing constraints, makes it impossible to precisely foresee the consequences of the decisions made. In order to analyse this sphere of economic agent's activity, it is deemed necessary to apply the tool ware of probability theory, decision science and management theory methods. Multifaceted and complex character of challenges, that are faced by the companies involved in international trade resulted in a situation, when decision making aimed to enhance the efficiency of the companies' operation in the global scale, has become the issue of multiple criteria optimisation without an unambiguous answer.

The best solution for a multiple criteria problem may be allocated in the sphere of criteria compromises used to analyse the international trade of a company and the constraints following its research, since the best alternative should meet them all at the same time. Efficiency management of international trade is viewed as a multiple criteria problem of the search of alternatives for its development in the segment of economy for the long term perspective, characterised by the multiplicity of optimality principles. It would be reasonable to form the optimality principles within a multiple criteria problem organized as either "... certain orderings..." [3] based on numerous alternatives, or utility functions, or functional from utility functions subject to maximisation. Should a decision maker select certain criteria for efficiency analysis of international trade, the best alternative becomes a very challenging issue. This is due to several reasons, one of them being inadequacy of current situation estimates in the sphere of international trade.

\footnotetext{
${ }^{1}$ Olga Martyanova, Candidate of Economic Sciences, doctoral candidate The Orel State University of Economics and Trade, Russia.
} 
It makes no economic sense to identify inaccurate estimates of the international trade, regardless of its nature, with randomness. We argue, that there is a distinction between randomness and fuzziness, which act as the core sources of inaccuracy in decision making in relation to export/import business processes. Under decision fuzziness of the international trade carried out by a company we deem the sort of inaccuracy related to fuzzy sets "... i.e. classes where no sharp boundaries can be drawn between the elements pertaining to this class and those allocated outside of it..." [2, p. 172]. Thus, randomness is attributed to uncertainty, determined by whether the international trade business process undergoing analysis belongs to the clearly defined set or not, while in fuzziness there may be different gradations of membership degree, including intermediate ones. For these reasons the problem of analysis of transactions performed by a company involved in international trade may be represented as follows: [5, p. 2]: $f(x, y, z) \rightarrow \min , x \in X, y \in Y, z \in Z$,

where $x$-is the vector of controlled factors,

$y$ - is the vector of random factors,

$Z$ - is the vector of fuzzy factors,

$X, Y, Z$ - refer to the subsets of certain vector spaces, which form a finite dimensional optimisation problem, provided that these spaces are bounded.

Driven by fuzziness, a decision maker is forced to use fuzzy sets when analysing the company's efficiency. These sets represent a class of objects where no sharp boundary can be drawn between the elements pertaining to this class and those allocated outside of it. Regardless of the boundary fuzziness of the set, it can be clearly defined by the object correlation with the number ranging from 0 to 1 , characterising the degree of its affiliation to the given set.

Currently the companies involved in international trade are characterized by the use of two criteria as a maximum, based on the calculation of a few coefficients. These are applied when selecting the best foreign supplier or foreign buyer, optimal provisions of foreign trade contract, making subjective decisions without taking into consideration the factors pertaining to the quality aspect of a company's international trade. However, the ever increasing demand for the instrumentation of management efficiency of a company's international trade and on the system of implementation mechanisms of the adopted strategies of the foreign trade necessitate the use of multiple criteria analysis along with a unified generalized indicator characterising the economic efficiency of a business entity involved in international trade as a whole taking into consideration all the partial criteria. This exactly is demanded by the contemporary ideology of a systems' approach when analysing the international trade of a business entity in general and foreign buyers in particular.

The problem of the choice of buyers in the sphere of international trade is an urgent issue for business entities not so much due to their growing number, but because of the necessity to run efficiently on the global market. This is deemed possible, provided that such important factors as the contractor's reputation, his/her financial position, purchase amount, supply needs exercised by buyers are taken into consideration. This issue is also relevant for business enti- 
ties undergoing tax control measures aimed to confirm the fact of gaining an unjustified tax benefit. This way the tax authorities may request the documents justifying the choice of both, national and international buyers, as well as data related to the activity of a business entity when selecting a contractor. The Federal Tax Service of Russia reminded business entities of this right is exercised by the tax authorities in a letter from 23.03.2017 о ЕД-5-9/547@ [1].

In order to analyse the fuzziness occurring when a company performs its international trade, it is deemed appropriate to apply the apparatus of a fuzzyset theory. This theory allows formulate the problem as the choice of the best alternative (a foreign customer) using a number of criteria including BellmanZadeh approach [2, p. 187]. This approach may be combined with Saaty's method of analytic hierarchies [6, p.26-28], thus, enabling to find the degrees of membership for the fuzzy set elements using paired comparison.

Saaty's method presumes building a matrix of paired comparisons and checking its consistency. If the matrix of paired comparison is consistent, then it develops eigenvector, defining the membership degree. Otherwise, it is necessary to make paired comparison again, which hinders putting this method into practice, since the procedure of building the matrix of paired comparison is very labour intensive. In order to eliminate this constraint, it is recommended to apply a multiple criteria analysis of a foreign customer using the intersection of Bellman-Zadeh approach and Saaty's method. This allows making an optimal solution by comparing ratios obtained by calculation with the worst alternative from all the available ones by the least important criterion. Therefore, this approach may be called as the worst alternative method.

Let us introduce the following notation:

$\boldsymbol{B}=\left\{b_{1}, b_{2}, \ldots, b_{n}\right\}-$ a set of foreign customers;

$\boldsymbol{K}=\left\{k_{1}, k_{2}, \ldots, k_{m}\right\}-$ a set of criteria.

The application of a vector criteria in the new model of foreign customers' analysis is driven by the complexity of decision making under uncertainty. In order to find the solution to our problem when all the possible outcomes are estimated using a vector criteria, we will refer to the scalarization method. In order to implement the selected scalarization method we will choose the convolution of criteria, since the values of the parameters calculated by every criterion selected by a business entity, can be expressed as one unit [4, p. 8]. The integrated assessment of $\mathrm{i}$-th alternative is obtained as a result of convolution, taking the result vector of its estimates $\boldsymbol{t}_{i}=\left\{t_{i 1}, t_{i 2}, \ldots, t_{i m}\right\}$ by every $k_{j}$ criterion to $T_{i}$ scalar. As a result of calculation we will obtain a set of estimates $T=\left\{T_{1}, T_{2}, \ldots, T_{n}\right\}$. The best estimate corresponds to the best alternative.

Thus, every criterion $k_{j}$ can be viewed as a fuzzy set $\widehat{\boldsymbol{k}}_{j}$ specified based on a universal set of foreign buyers $\boldsymbol{B}=\left\{b_{1}, b_{2}, \ldots, b_{n}\right\}$ and may be presented as follows [7, p. 4]:

$$
\widehat{\boldsymbol{k}}_{j}=\left\{\frac{\mu_{k_{1}}\left(b_{1}\right)}{b_{1}} ; \frac{\mu_{k_{2}}\left(b_{2}\right)}{b_{2}} ; \ldots ; \frac{\mu_{k_{m}}\left(b_{n}\right)}{b_{n}}\right\}, j=1, \ldots, m ; i=1, \ldots, n,(1)
$$


where $\mu_{k_{m}}\left(b_{n}\right)$ - is the membership degree of element $b_{i}$ in relation to the fuzzy set $\widehat{\boldsymbol{k}}_{\boldsymbol{j}}$, ranging from 0 to 1 , i.e. the parameter $\mu_{k_{m}}\left(b_{n}\right)$ evaluates option $b_{i}$ by criterion $k_{j}[2$, p. 176].

Since the parameter $\mu_{k_{m}}\left(b_{n}\right)$ estimates alternative $b_{i}$ by criterion $k_{j}$, then the higher the degree of membership of $\mu_{k_{m}}\left(b_{n}\right)$ is, the better the option $b_{i}$ by criterion $k_{j}$ shall be. Numerous alternatives, each of which is evaluated by the referent criteria from the set $\boldsymbol{K}=\left\{k_{1}, k_{2}, \ldots, k_{m}\right\}$, should at the same time meet a set of constraints $\boldsymbol{R}=\left\{r_{1}, r_{2}, \ldots, r_{l}\right\}$, representing fuzzy sets $\hat{\boldsymbol{r}}_{z}$ with their own membership degrees of the following type[7, p. 4]:

$$
\hat{\boldsymbol{r}}_{z}=\left\{\frac{\mu_{r_{1}}\left(b_{1}\right)}{b_{1}} ; \frac{\mu_{r_{2}}\left(b_{2}\right)}{b_{2}} ; \ldots ; \frac{\mu_{r_{l}}\left(b_{n}\right)}{b_{n}}\right\}, z=1, \ldots, l .
$$

The determined criteria and constraints, as applicable to set $\boldsymbol{B}=$ $\left\{b_{1}, b_{2}, \ldots, b_{n}\right\}$, enable to generate a fuzzy set of final estimates alternatives $\widehat{\boldsymbol{T}}$, which can be represented as follows [7, p. 5]:

$$
\widehat{\boldsymbol{T}}=\left\{\frac{\mu_{T}\left(b_{1}\right)}{b_{1}} ; \frac{\mu_{T}\left(b_{2}\right)}{b_{2}} ; \ldots ; \frac{\mu_{T}\left(b_{n}\right)}{b_{n}}\right\} .
$$

Thus, the membership degree of a fuzzy set $\mu_{T}\left(b_{n}\right)$ shows to what extent this evaluation meets fuzzy goals and fuzzy constraints.

In order to determine the best foreign customer, we will refer to Bellmann-Zadeh approach. According to this approach the alternative, which meets all the criteria and constraints to the most extent at the same time is the optimal one. The solution is represented as the intersection of fuzzy sets, constraints and partial criteria as follows [2, p. 187]:

$$
b_{\text {optimal }} \in \widehat{\boldsymbol{T}}=\widehat{\boldsymbol{r}}_{1} \cap \widehat{\boldsymbol{r}}_{2} \cap \ldots \cap \widehat{\boldsymbol{r}}_{l} \cap \widehat{\boldsymbol{k}}_{1} \cap \widehat{\boldsymbol{k}}_{2} \cap \ldots \cap \widehat{\boldsymbol{k}}_{m} \text {. (4) }
$$

The theory of fuzzy sets defines the intersection of fuzzy sets as the smallest fuzzy set contained in every of the intersecting sets, which enables determining the membership degree (function), e.g. for $r_{1} \cap r_{2}$ represented as the following equation [2, p. 179]:

$$
\mu_{r_{1} \cap r_{2}}(b)=\min \left(\mu_{r_{1}}(b), \mu_{r_{2}}(b)\right), b \in \boldsymbol{B}, \text { (5) }
$$

where $\min \left(r_{1}, r_{2}\right)=r_{1}$, if $r_{1} \leq r_{2}$, and $\min \left(r_{1}, r_{2}\right)=r_{2}$, if $r_{1}>r_{2}$.

If we substitute symbol min with the logic operation of conjunction $\&$, then the formula (5) for the membership functions may be represented as follows [2, p. 179]:

$$
\mu_{r_{1} \cap r_{2}}=\mu_{r_{1}} \& \mu_{r_{2}} \cdot(6)
$$

In this case the equation (4) will be represented as follows:

$$
\mu_{T}=\mu_{r_{1}} \& \mu_{r_{2}} \& \ldots \& \mu_{r_{l}} \& \mu_{k_{1}} \& \mu_{k_{2}} \text { (7) }
$$

Should criteria $K$ and constraints $R$ be of different importance, we believe it is necessary to introduce the coefficients of their relative importance $\sigma_{j} \in$ $(0 ; 1), j=1, \ldots, m$ and $\gamma_{z} \in(0 ; 1), z=1, \ldots, l$, respectively, meeting the following condition [2, p. 191]:

$$
\sum_{z=1}^{l} \gamma_{z}+\sum_{j=1}^{m} \sigma_{j}=1 \text {. (8) }
$$


In this case the membership degree of final estimate of alternatives will be defined as follows [7, p. 5]:

$$
\mu_{T}=\left(\mu_{r_{1}}\right)^{\gamma_{1}} \&\left(\mu_{r_{2}}\right)^{\gamma_{2}} \& \ldots \&\left(\mu_{r_{l}}\right)^{\gamma_{l}} \&\left(\mu_{k_{1}}\right)^{\sigma_{1}}(9)
$$

In accordance with the rule of carrying out operations with fuzzy sets described above (5), we will transfer expression (4) into the equation as follows:

$$
\widehat{\boldsymbol{T}}=\left\{\frac{\min _{j=1, m}\left(\mu_{k_{j}}\left(b_{1}\right), \mu_{r_{z}}\left(b_{1}\right)\right)}{b_{1}} ; \ldots ; \frac{\min _{j=1, m}\left(\mu_{k_{j}}\left(b_{n}\right), \mu_{r_{z}}\left(b_{n}\right)\right)}{b_{n}}\right\}
$$

The alternative with the highest membership degree [2, p. 190] should be chosen as the next best alternative of $b_{\text {optimal }}$ from the obtained fuzzy set of final alternative estimates $\widehat{\boldsymbol{T}}(10)$ :

or

$$
T\left(b_{\text {optimal }}\right)=\underset{b_{i}}{\operatorname{argmax}}\left(\mu_{T}\left(b_{1}\right), \mu_{T}\left(b_{2}\right), \ldots, \mu_{T}\left(b_{n}\right)\right),(
$$

else

$$
T\left(b_{\text {optimal }}\right)=\underset{b_{i}}{\operatorname{argmax}}\left(\frac{\min _{j=1, m}\left(\mu_{k_{j}}\left(b_{1}\right), \mu_{r_{z}}\left(b_{1}\right)\right)}{b_{1}} ; \ldots ; \frac{\min _{j=1, m}\left(\mu_{k_{j}}\left(b_{n}\right), \mu_{r_{z}}\left(b_{n}\right)\right)}{b_{n}}\right)
$$

Should criteria and constraints have different degrees of importance, then the membership function for the fuzzy set of final alternative evaluation $\mu_{T}\left(b_{i}\right)$ will be determined as follows:

$$
\mu_{T}\left(b_{i}\right)=\min _{\substack{j=1, m \\ z=1, l}}\left[\left(\mu_{k_{j}}\left(b_{i}\right)\right)^{\sigma_{j}},\left(\mu_{r_{z}}\left(b_{i}\right)\right)^{\gamma_{z}}\right], i=1, \ldots, n
$$

Thus, the equation (12) will be represented as follows:

$$
\underset{b_{i}}{\operatorname{argmax}}\left(\frac{\min _{j=1, m}\left[\left(\mu_{k_{j}}\left(b_{1}\right)\right)^{\sigma_{j}},\left(\mu_{r_{z}}\left(b_{1}\right)\right)^{\gamma_{z}}\right]}{b_{1}} ; \ldots ; \frac{\min _{\substack{\gamma_{1}, m \\ z=1, l}}\left[\left(\mu_{k_{j}}\left(b_{n}\right)\right)^{\sigma_{j}},\left(\mu_{r_{z}}\left(b_{n}\right)\right)^{\gamma_{z}}\right]}{b_{n}}\right) .
$$

When comparing international buyers using one criterion, we rely on expert evaluation, therefore, in order to determine the membership functions, it is deemed relevant to use a paired comparison method of alternative options along with Saaty's 9-point scale [6, p. 53]. To do so we first defined the level of advantage $\left(m_{i j}\right)$ of one buyer $\left(b_{i}\right)$ over another $\left(b_{j}\right)(i, j=1, k)$, evaluated according to the scale provided in Table 1 . The system of paired comparison results in antisymmetric $\left(m_{i j}=1 / m_{j i} ; i, j=1, k\right)$ and diagonal matrix reflecting the opinion of a decision maker in relation to the importance of different criteria. 
Table 1

Levels of advantage of international buyers evaluated based on Saaty's scale

\begin{tabular}{|l|c|}
\hline Characteristics of the level of advantage of $b_{i}$ over $b_{j}$ & Evaluation scale \\
\hline Absent & 1 \\
\hline Almost weak & 2 \\
\hline Weak & 3 \\
\hline Almost significant & 4 \\
\hline Significant & 5 \\
\hline Almost obvious & 6 \\
\hline Obvious & 7 \\
\hline Almost absolute & 8 \\
\hline Absolute & 9 \\
\hline
\end{tabular}

The membership functions $\mu\left(b_{i}\right)$ are deemed equal to the corresponding coordinates of eigenvector $w_{i}$ of the paired comparison matrix $\boldsymbol{M}$ and are represented as follows $[7$, p. 5]:

$$
\mu\left(b_{i}\right)=w_{i}, i=1, k .(15)
$$

The eigenvector is derived from the following system of equations $[6, \mathrm{p}$. 50]:

$$
\left\{\begin{array}{c}
\boldsymbol{M} \times \boldsymbol{w}=n_{\max } \times \boldsymbol{w} \\
w_{1}+\cdots+w_{n}=1
\end{array}\right.
$$

Where $n_{\max }$ - is the maximum value of matrix $\boldsymbol{M}$.

Let us take a look at the practical implementation of the method of decision making under uncertainty using Bellman-Zadeh approach and take the following stages:

Stage 1: Select the criteria for alternative evaluation.

Stage 2: Carry out a comparative evaluation of the alternatives in accordance with the selected criteria in the linguistic form.

Stage 3: Generate paired comparison matrices for the alternatives compatible with the evaluation carried out in the linguistic form.

Stage 4: Evaluate the membership degree of alternatives attributed to the fuzzy sets of the selected criteria.

Stage 5: Generate fuzzy sets of criteria compatible with the obtained membership degrees of alternatives.

Stage 6: Carry out paired comparisons of the selected criteria in the linguistic form.

Stage 7: Generate the matrix of paired comparisons of the criteria based on their evaluation carried out in the linguistic form.

Stage 8: Determine the coefficients of relative importance of criteria using the results of their evaluation based on paired comparisons.

Stage 9: Find the fuzzy sets of the criteria depending on the degree of their importance.

Stage 10: Form a fuzzy set of final estimates of alternatives.

Stage 11: Choose the best alternative according to Bellman-Zadeh approach that would meet all the criteria to the most extent.

The exporter of upmarket beer should choose the best buyer from the array of economic entities abroad including the distributor's organization focusing on upmarket product $\left(b_{1}\right)$, international restaurant network $\mathrm{HoReCa}$ with its 
branched chain in different countries worldwide $\left(b_{2}\right)$, and a beerhouse $\left(b_{3}\right)$. The exporter compares the foreign buyers using the following criteria:

$\mathrm{k}_{1}$ - Buyer's image in the business environment;

$\mathrm{k}_{2}$-Range of the purchased product;

$\mathrm{k}_{3}$-Extension of the sales network, number of retail outlets;

$\mathrm{k}_{4}$-Purchase volume;

$\mathrm{k}_{5}$-Financial condition of the buyer;

$\mathrm{k}_{6}$-Supply conditions and demands (merchandise return and exchange, cooperation costs, crediting options);

$\mathrm{k}_{7}$-Price strategy;

$\mathrm{k}_{8}$-Infrastructure (development of transportation routes, availability and condition of warehouses).

The alternatives (international buyers) were linguistically evaluated by a decision maker. The results of this evaluation are provided in Table 2 .

Table 2

Comparative evaluation of alternatives by the selected criteria

\begin{tabular}{|c|c|c|c|}
\hline Criterion & Compared alternatives & Charact & ics of alternative advantage \\
\hline \multirow{3}{*}{$k_{1}$} & $b_{1}$ and $b_{2}$ & $b_{1}$ over $b_{2}$ & almost significant \\
\hline & $b_{1}$ and $b_{3}$ & $b_{1}$ over $b_{3}$ & evident \\
\hline & $b_{2}$ and $b_{3}$ & $b_{2}$ over $b_{3}$ & weak \\
\hline \multirow{3}{*}{$k_{2}$} & $b_{2}$ and $b_{1}$ & $b_{2}$ over $b_{1}$ & absolute \\
\hline & $b_{3}$ and $b_{1}$ & $b_{3}$ over $b_{1}$ & almost significant \\
\hline & $b_{2}$ and $b_{3}$ & $b_{2}$ over $b_{3}$ & almost significant \\
\hline \multirow{3}{*}{$k_{3}$} & $b_{1}$ and $b_{2}$ & $b_{1}$ over $b_{2}$ & significant \\
\hline & $b_{1}$ and $b_{3}$ & $b_{1}$ over $b_{3}$ & almost weak \\
\hline & $b_{3}$ and $b_{2}$ & $b_{3}$ over $b_{2}$ & almost weak \\
\hline \multirow{3}{*}{$k_{4}$} & $b_{1}$ and $b_{2}$ & $b_{1}$ over $b_{2}$ & almost weak \\
\hline & $b_{1}$ and $b_{3}$ & $b_{1}$ over $b_{3}$ & almost significant \\
\hline & $b_{3}$ and $b_{2}$ & $b_{3}$ over $b_{2}$ & almost weak \\
\hline \multirow{3}{*}{$k_{5}$} & $b_{1}$ and $b_{2}$ & $b_{1}$ over $b_{2}$ & evident \\
\hline & $b_{1}$ and $b_{3}$ & $b_{1}$ over $b_{3}$ & weak \\
\hline & $b_{3}$ and $b_{2}$ & $b_{3}$ over $b_{2}$ & absent \\
\hline \multirow{3}{*}{$k_{6}$} & $b_{2}$ and $b_{1}$ & $b_{2}$ over $b_{1}$ & almost significant \\
\hline & $b_{3}$ and $b_{1}$ & $b_{3}$ over $b_{1}$ & absolute \\
\hline & $b_{3}$ and $b_{2}$ & $b_{3}$ over $b_{2}$ & almost weak \\
\hline \multirow{3}{*}{$k_{7}$} & $b_{1}$ and $b_{2}$ & $b_{1}$ over $b_{2}$ & weak \\
\hline & $b_{3}$ and $b_{1}$ & $b_{3}$ over $b_{1}$ & almost significant \\
\hline & $b_{3}$ and $b_{2}$ & $b_{3}$ over $b_{2}$ & evident \\
\hline \multirow{3}{*}{$k_{8}$} & $b_{2}$ and $b_{1}$ & $b_{2}$ over $b_{1}$ & almost absolute \\
\hline & $b_{2}$ and $b_{3}$ & $b_{2}$ over $b_{3}$ & weak \\
\hline & $b_{3}$ and $b_{1}$ & $b_{3}$ over $b_{1}$ & almost evident \\
\hline
\end{tabular}


Matrices of paired alternative comparisons, corresponding to their evaluation, represented in the linguistic form run as follows:

$$
\begin{aligned}
\boldsymbol{M}\left(k_{1}\right) & =\left(\begin{array}{ccc}
1 & 4 & 7 \\
1 / 4 & 1 & 3 \\
1 / 7 & 1 / 3 & 1
\end{array}\right) \\
\boldsymbol{M}\left(k_{2}\right) & =\left(\begin{array}{ccc}
1 & 1 / 9 & 1 / 4 \\
9 & 1 & 4 \\
4 & 1 / 4 & 1
\end{array}\right) \\
\boldsymbol{M}\left(k_{3}\right) & =\left(\begin{array}{ccc}
1 & 5 & 2 \\
1 / 5 & 1 & 1 / 6 \\
1 / 2 & 6 & 1
\end{array}\right) \\
\boldsymbol{M}\left(k_{4}\right) & =\left(\begin{array}{ccc}
1 & 6 & 4 \\
1 / 6 & 1 & 1 / 2 \\
1 / 4 & 2 & 1
\end{array}\right)
\end{aligned}
$$

$$
\begin{gathered}
\boldsymbol{M}\left(k_{5}\right)=\left(\begin{array}{ccc}
1 & 7 & 3 \\
1 / 7 & 1 & 1 \\
1 / 3 & 1 & 1
\end{array}\right) \\
\boldsymbol{M}\left(k_{6}\right)=\left(\begin{array}{ccc}
1 & 1 / 4 & 1 / 9 \\
4 & 1 & 1 / 2 \\
9 & 2 & 1
\end{array}\right) \\
\boldsymbol{M}\left(k_{7}\right)=\left(\begin{array}{ccc}
1 & 3 & 1 / 4 \\
1 / 3 & 1 & 1 / 7 \\
4 & 7 & 1
\end{array}\right) \\
\boldsymbol{M}\left(k_{8}\right)=\left(\begin{array}{ccc}
1 & 1 / 8 & 1 / 6 \\
8 & 1 & 3 \\
6 & 1 / 3 & 1
\end{array}\right)
\end{gathered}
$$

According to the method of analytic hierarchies it is necessary to check whether the obtained matrices of paired comparisons are consistent. For this purpose, let us calculate the coefficient of consistency $C R$, presenting information on the degree of consistency discrepancies viewed as follows [6, p. 25]:

$$
C R=\frac{C I}{R I},(17)
$$

where $C I=\frac{n_{\max }-n}{n-1}-$ consistency index of matrix $\boldsymbol{M}$; (18)

$n$ - number of elements in $i$-th line of matrix $\boldsymbol{M}$;

$n_{\text {max }}-$ maximum Eigen value of matrix $\boldsymbol{M}$;

$R I=\frac{1.98 \times(n-2)}{n}-$ stochastic coefficient of consistence of matrix $\boldsymbol{M}$. (19)

If $C R<0.1$, then inconsistency level is acceptable [6, p. 51], otherwise a decision maker has to check the elements of paired comparison $m_{i j}$ of matrix $\boldsymbol{M}$ in order to obtain a more consistent matrix. The results revealing the level of inconsistency of paired comparison matrices based on the selected criteria are provided in Table 3.

Table 3

Level of inconsistency of alternative comparison matrices based on criteria

\begin{tabular}{|c|c|c|c|c|}
\hline Matrix & $\begin{array}{c}\text { Consistency in- } \\
\text { dex of a matrix, }\end{array}$ & $\begin{array}{c}\text { Stochastic coeffi- } \\
\text { cient of matrix } \\
\text { consistency, } \\
R I\end{array}$ & $\begin{array}{c}\text { Coefficient of } \\
\text { matrix con- } \\
\text { sistency, } \\
C R\end{array}$ & $\begin{array}{c}\text { Level of matrix in- } \\
\text { consistency }\end{array}$ \\
\hline $\boldsymbol{M}\left(k_{j}\right)$ & $C I$ & 0.66 & 0.04 & Acceptable \\
\hline $\boldsymbol{M}\left(k_{1}\right)$ & 0.03 & 0.66 & 0.05 & Acceptable \\
\hline $\boldsymbol{M}\left(k_{2}\right)$ & 0.03 & 0.66 & 0.09 & Acceptable \\
\hline $\boldsymbol{M}\left(k_{3}\right)$ & 0.06 & 0.66 & 0.01 & Acceptable \\
\hline $\boldsymbol{M}\left(k_{4}\right)$ & 0.01 & 0.66 & 0.10 & Acceptable \\
\hline $\boldsymbol{M}\left(k_{5}\right)$ & 0.06 & 0.66 & 0.00 & Acceptable \\
\hline $\boldsymbol{M}\left(k_{6}\right)$ & 0.00 & 0.66 & 0.04 & Acceptable \\
\hline $\boldsymbol{M}\left(k_{7}\right)$ & 0.03 & 0.66 & 0.08 & Acceptable \\
\hline $\boldsymbol{M}\left(k_{8}\right)$ & 0.06 & & & \\
\hline
\end{tabular}

Due to the fact that the inconsistency level is acceptable for matrices, we will define the membership degree of alternatives in the fuzzy set of criteria $\mu_{k_{j}}\left(b_{i}\right)$. For this purpose we will solve a system (16) for every paired comparison matrix of alternatives taking expression (15) into consideration. The results of the calculations carried out are provided in Table 4. 
Table 4

Membership degree of alternatives in the fuzzy set of selected criteria

\begin{tabular}{|c|c|c|c|}
\hline \multirow{2}{*}{$\begin{array}{c}\text { Criterion } \\
\left(k_{j}\right)\end{array}$} & \multicolumn{3}{|c|}{ Membership degree $\mu_{k_{j}}\left(b_{i}\right)$ of alternatives } \\
\cline { 2 - 4 } & $b_{1}$ & $b_{2}$ & $b_{3}$ \\
\hline$k_{1}$ & 0.70 & 0.21 & 0.09 \\
\hline$k_{2}$ & 0.07 & 0.71 & 0.22 \\
\hline$k_{3}$ & 0.55 & 0.08 & 0.37 \\
\hline$k_{4}$ & 0.70 & 0.11 & 0.19 \\
\hline$k_{5}$ & 0.69 & 0.14 & 0.18 \\
\hline$k_{6}$ & 0.07 & 0.30 & 0.63 \\
\hline$k_{7}$ & 0.21 & 0.09 & 0.70 \\
\hline$k_{8}$ & 0.06 & 0.65 & 0.29 \\
\hline
\end{tabular}

The fuzzy sets of criteria $\widehat{\boldsymbol{k}}_{j}$, corresponding to the obtained degrees of memberships can be represented as follows:

$$
\begin{array}{ll}
\widehat{\boldsymbol{k}}_{1}=\left\{\frac{0.70}{b_{1}} ; \frac{0.21}{b_{2}} ; \frac{0.09}{b_{3}}\right\} ; & \widehat{\boldsymbol{k}}_{5}=\left\{\frac{0.69}{b_{1}} ; \frac{0.14}{b_{2}} ; \frac{0.18}{b_{3}}\right\} ; \\
\widehat{\boldsymbol{k}}_{2}=\left\{\frac{0.07}{b_{1}} ; \frac{0.71}{b_{2}} ; \frac{0.22}{b_{3}}\right\} ; & \widehat{\boldsymbol{k}}_{6}=\left\{\frac{0.07}{b_{1}} ; \frac{0.30}{b_{2}} ; \frac{0.63}{b_{3}}\right\} ; \\
\widehat{\boldsymbol{k}}_{3}=\left\{\frac{0.55}{b_{1}} ; \frac{0.08}{b_{2}} ; \frac{0.37}{b_{3}}\right\} ; & \widehat{\boldsymbol{k}}_{7}=\left\{\frac{0.21}{b_{1}} ; \frac{0.09}{b_{2}} ; \frac{0.70}{b_{3}}\right\} ; \\
\widehat{\boldsymbol{k}}_{4}=\left\{\frac{0.70}{b_{1}} ; \frac{0.11}{b_{2}} ; \frac{0.19}{b_{3}}\right\} ; & \widehat{\boldsymbol{k}}_{8}=\left\{\frac{0.06}{b_{1}} ; \frac{0.65}{b_{2}} ; \frac{0.29}{b_{3}}\right\}
\end{array}
$$

The results of criteria paired comparisons carried out in the linguistic

\begin{tabular}{|c|c|c|c|c|c|c|}
\hline \multicolumn{3}{|c|}{ Compared criteria } & \multicolumn{4}{|c|}{ Characteristics of the compared criteria } \\
\hline$k_{1}$ & and & $k_{2}$ & $k_{1}$ & over & $k_{2}$ & almost evident \\
\hline$k_{1}$ & and & $k_{3}$ & $k_{1}$ & over & $k_{3}$ & almost weak \\
\hline$k_{1}$ & and & $k_{5}$ & $k_{1}$ & over & $k_{5}$ & significant \\
\hline$k_{1}$ & and & $k_{7}$ & $k_{1}$ & over & $k_{7}$ & almost significant \\
\hline$k_{1}$ & and & $k_{8}$ & $k_{1}$ & over & $k_{8}$ & almost weak \\
\hline$k_{3}$ & and & $k_{2}$ & $k_{3}$ & over & $k_{2}$ & evident \\
\hline$k_{3}$ & and & $k_{5}$ & $k_{3}$ & over & $k_{5}$ & evident \\
\hline$k_{3}$ & and & $k_{6}$ & $k_{3}$ & over & $k_{6}$ & almost weak \\
\hline$k_{3}$ & and & $k_{7}$ & $k_{3}$ & over & $k_{7}$ & weak \\
\hline$k_{3}$ & and & $k_{8}$ & $k_{3}$ & over & $k_{8}$ & almost weak \\
\hline$k_{4}$ & and & $k_{1}$ & $k_{4}$ & over & $k_{1}$ & almost weak \\
\hline$k_{4}$ & and & $k_{2}$ & $k_{4}$ & over & $k_{2}$ & absolute \\
\hline$k_{4}$ & and & $k_{3}$ & $k_{4}$ & over & $k_{3}$ & almost weak \\
\hline$k_{4}$ & and & $k_{5}$ & $k_{4}$ & over & $k_{5}$ & almost absolute \\
\hline$k_{4}$ & and & $k_{6}$ & $k_{4}$ & over & $k_{6}$ & almost significant \\
\hline$k_{4}$ & and & $k_{7}$ & $k_{4}$ & over & $k_{7}$ & weak \\
\hline$k_{4}$ & and & $k_{8}$ & $k_{4}$ & over & $k_{8}$ & evident \\
\hline$k_{5}$ & and & $k_{2}$ & $k_{5}$ & over & $k_{2}$ & almost weak \\
\hline$k_{6}$ & and & $k_{1}$ & $k_{6}$ & over & $k_{1}$ & almost weak \\
\hline$k_{6}$ & and & $k_{2}$ & $k_{6}$ & over & $k_{2}$ & almost evident \\
\hline$k_{6}$ & and & $k_{5}$ & $k_{6}$ & over & $k_{5}$ & significant \\
\hline$k_{6}$ & and & $k_{7}$ & $k_{6}$ & over & $k_{7}$ & weak \\
\hline$k_{6}$ & and & $k_{8}$ & $k_{6}$ & over & $k_{8}$ & almost weak \\
\hline$k_{7}$ & and & $k_{2}$ & $k_{7}$ & over & $k_{2}$ & almost weak \\
\hline$k_{7}$ & and & $k_{5}$ & $k_{7}$ & over & $k_{5}$ & weak \\
\hline$k_{8}$ & and & $k_{2}$ & $k_{8}$ & over & $k_{2}$ & weak \\
\hline$k_{8}$ & and & $k_{5}$ & $k_{8}$ & over & $k_{5}$ & almost weak \\
\hline$k_{8}$ & and & $k_{7}$ & $k_{8}$ & over & $k_{7}$ & weak \\
\hline
\end{tabular}
form are presented in Table 5 .

Table 5

Comparative estimate of criteria selected by the exporter 
The system of paired comparisons results in antisymmetrical diagonal matrix, which can be presented as follows:

$$
\boldsymbol{M}=\left(\begin{array}{cccccccc}
1 & 6 & 2 & 1 / 2 & 5 & 1 / 2 & 4 & 2 \\
1 / 6 & 1 & 1 / 7 & 1 / 9 & 1 / 2 & 1 / 6 & 1 / 2 & 1 / 3 \\
1 / 2 & 7 & 1 & 1 / 2 & 7 & 2 & 3 & 2 \\
2 & 9 & 2 & 1 & 8 & 4 & 3 & 7 \\
1 / 5 & 2 & 1 / 7 & 1 / 8 & 1 & 1 / 5 & 1 / 3 & 1 / 2 \\
2 & 6 & 1 / 2 & 1 / 4 & 5 & 1 & 3 & 2 \\
1 / 4 & 2 & 1 / 3 & 1 / 3 & 3 & 1 / 3 & 1 & 1 / 3 \\
1 / 2 & 3 & 1 / 2 & 1 / 7 & 2 & 1 / 2 & 3 & 1
\end{array}\right)(20)
$$

Matrix (20) verification in terms of its consistency proves that $C R=$ $0.061<0.1$, therefore the level of inconsistency of matrix $\boldsymbol{M}$ is acceptable.

Since the criteria are of different importance, it is necessary to calculate the coefficients of relative importance for the criteria $\sigma_{j}$. For this purpose we will solve the system of equations (16) for matrix (20), which resulted in the following values:

$$
\begin{array}{ll}
\sigma_{1}=0.1701 ; & \sigma_{5}=0.0320 \\
\sigma_{2}=0.0246 ; & \sigma_{6}=0.1503 \\
\sigma_{3}=0.1678 ; & \sigma_{7}=0.0585 \\
\sigma_{4}=0.3169 ; & \sigma_{8}=0.0797
\end{array}
$$

The analysis of the coefficients of relative importance of the criteria proves that criterion $k_{4}$ is of utmost importance.

We will determine fuzzy sets of criteria based on the alternatives available $\left(k_{j}^{\sigma_{j}}\right)$, using expression (13) and the obtained values of the coefficients of relative importance of criteria $\sigma_{j}$. The calculations carried out enabled us to determine the following fuzzy sets:

$$
\begin{aligned}
& k_{6}^{\sigma_{6}}=\left\{\frac{0.07^{0.1503}}{b_{1}} ; \frac{0.30^{0.1503}}{b_{2}} ; \frac{0.63^{0.1503}}{b_{3}}\right\}=\left\{\frac{0.674}{b_{1}} ; \frac{0.835}{b_{2}} ; \frac{0.932}{b_{3}}\right\} ; \\
& k_{7}^{\sigma_{7}}=\left\{\frac{0.21^{0.0585}}{b_{1}} ; \frac{0.09^{0.0585}}{b_{2}} ; \frac{0.70^{0.0585}}{b_{3}}\right\}=\left\{\frac{0.914}{b_{1}} ; \frac{0.866}{b_{2}} ; \frac{0.979}{b_{3}}\right\} \\
& k_{8}^{\sigma_{8}}=\left\{\frac{0.06^{0.0797}}{b_{1}} ; \frac{0.65^{0.0797}}{b_{2}} ; \frac{0.29^{0.0797}}{b_{3}}\right\}=\left\{\frac{0.803}{b_{1}} ; \frac{0.966}{b_{2}} ; \frac{0.906}{b_{3}}\right\}
\end{aligned}
$$

The intersection of the obtained fuzzy sets of criteria, according to expression (10), enables to define the values of the membership degrees for a fuzzy set of the final evaluation of alternatives $\mu_{T}\left(b_{i}\right)$, which equal the following:

$$
\begin{aligned}
& \mu_{T}\left(b_{1}\right)=\min \{0.941 ; 0.936 ; 0.903 ; 0.893 ; 0.988 ; 0.674 ; 0.914 ; 0.803\}=0.674 ; \\
& \mu_{T}\left(b_{2}\right)=\min \{0.769 ; 0,992 ; 0.661 ; 0.492 ; 0.938 ; 0.835 ; 0.866 ; 0.966\}=0.492 ; \\
& \mu_{T}\left(b_{3}\right)=\min \{0.658 ; 0.963 ; 0.846 ; 0.594 ; 0.946 ; 0.932 ; 0.979 ; 0.906\}=0.594 .
\end{aligned}
$$

Thus, the fuzzy set of final evaluations will have the following representation:

$$
\widehat{\boldsymbol{T}}=\left\{\frac{0.674}{b_{1}} ; \frac{0.492}{b_{2}} ; \frac{0.594}{b_{3}}\right\}
$$


By means of applying expression (14) to the membership degrees of a fuzzy set (26), we will determine the best (most optimal) alternative ( $\left.b_{\text {optimal }}\right)$ in accordance with Bellman-Zadeh approach:

$$
\mu_{T}\left(b_{\text {optimal }}\right)=\max \{0.674 ; 0.492 ; 0.594\}=0.674=\mu_{T}\left(b_{1}\right),
$$

i.e. $T=b_{1}$.

Thus, the distributor, dealing with upmarket products, meets all the criteria chosen by the exporter to evaluate foreign buyers taking into consideration the degree of their importance.

Systematization of the analysis results allows us to draw the following conclusions:

1. Extending the apparatus of fuzzy sets with the method of analytic hierarchies and decision making based on Bellman-Zadeh approach enable us to generate a tool ware which makes it possible to carry out a multiple criteria analysis of international trade of a business entity based on the unified criterion, developed by the company in order to select the best buyer.

2. The suggested method enables the exporter to select the optimal international buyer by means of employing a particular criterion as well. Efficiency of operation, being a complex characteristic, may act as this particular criterion for multiple alternatives. Therefore, the developed method for decision making under economic uncertainties elaborates the theoretical model of the contents characteristics of strategic management of the company's efficiency in international trade, based on methodological provisions of economic analysis and the theory of fuzzy sets.

3. The model of decision making based on Bellman-Zadeh approach makes it possible to develop the concept of efficiency management of the company's foreign trade using contemporary approaches to the analysis of its foreign trade strategy and taking into consideration the particularities of the company's operation in the sphere of foreign trade.

4. The developed model of foreign buyers' evaluation has a number of advantages. First of all, it presumes the bulk of work on the stage of evaluation criteria identification applicable to contractors, which is the advantage of this method, since the detailed factor determination undergoing evaluation makes it possible to carry out a precise analysis of the available alternatives. Secondly, it enables choosing a decision viewed as a fuzzy set among alternatives, provided that the priorities of a decision maker in terms of implementation of this or that option remain unknown. Thirdly, the suggested method based on Bellman-Zadeh approach is universal, as it enables selecting international buyers as well as an optimal buyer on the domestic market. The method allows carrying out a quality analysis of all risk factors associated with the choice of a trading partner. A large amount of calculation is obviously a drawback 
of the suggested method. However, it can be easily eliminated by computerized calculations.

\section{References}

[1] About identification of circumstances of unreasonable tax benefit [Electronic resource]: letter of Federal Tax Service of Russia of 23.03.2017 № ED-5-9/547@. Access from the legal-reference ConsultantPlus system.

[2] Bellman R. Questions of the analysis and procedure of decision making [Text]: collection of translations // R. Bellman, L. Zade; under edition of the candidate of physical and mathematical sciences I. F. Shakhnov. - M.: Mir publishing house, 1976. - 215 pages.

[3] Hadzaragova E. A. Research of a skalyarization of vector estimates in multicriteria optimization tasks [Electronic resource] / E. A. Hadzaragova // Urgent problems of humanitarian and natural sciences. - 2015. - № 3-3. - URL: http://publikacia.net/archive $/ 2015 / 3 / 3 / 3$.

[4] Konnov I.V. About a skalyarization of vector tasks of optimization type [Text] / I.V. Konnov // News of higher educational institutions. Mathematics. - 2012. - № 9. - Page 8-18.

[5] Pisaruk N. N. Operations research [Text] / N. N. Pisaruk. - Minsk: Belarusian State University, 2015. - 304 pages.

[6] Saati T. Decision making. Method of the analysis of hierarchies [Text]: T. Saati; the translation from the English R. G. Vachnadze. - M.: Radio and communication, 1993. 278 pages.

[7] Zezin V. G. Metodiko-programmny the choice module like system of initiation of pyromeans of a missile system in the conditions of essential uncertainty [Text] / V. G. Zezin, M. I. Reshetnikov // Messenger of the Izhevsk State technical university of M. T. Kalashnikov. - 2014. - № 4(64). - Page 4-7. 